\title{
Superstability and Solution of The Pexiderized Trigonometric Functional Equation
}

\author{
Gwang Hui Kim \\ Department of Mathematics, Kangnam University Yongin, Gyeonggi, 16979, Korea
}

Received January 16, 2020; Revised April 16, 2020; Accepted April 27, 2020

\begin{abstract}
Copyright $\odot 2020$ by authors, all rights reserved. Authors agree that this article remains permanently open access under the terms of the Creative Commons Attribution License 4.0 International License
\end{abstract}

\begin{abstract}
The present work continues the study for the superstability and solution of the Pexider type functional equation $f(x+y)+g(x-y)=\lambda \cdot h(x) k(y)$, which is the mixed functional equation represented by sum of the sine, cosine, tangent, hyperbolic trigonometric, and exponential functions. The stability of the cosine (d'Alembert) functional equation and the Wilson equation was researched by many authors: Baker [7], Badora [5], Kannappan [14], Kim ([16, 19]), and Fassi, etc [11]. The stability of the sine type equations was researched by Cholewa [10], Kim ([18], [20]). The stability of the difference type equation $f(x+y)-f(x-y)=\lambda \cdot h(x) k(y)$ for the above equation was studied by Kim ([21], [22]). In this paper, we investigate the superstability of the sine functional equation and the Wilson equation from the Pexider type difference functional equation $f(x+y)-g(x-y)=\lambda \cdot h(x) k(y)$, which is the mixed equation represented by the sine, cosine, tangent, hyperbolic trigonometric functions, and exponential functions. Also, we obtain additionally that the Wilson equation and the cosine functional eqaution in the obtained results can be represented by the composition of a homomorphism. In here, the domain $(G,+)$ of functions $f, g, h, k: G \rightarrow A$ is a noncommutative semigroup (or 2-divisible Abelian group), and $A$ is an unital commutative normed algebra with unit $1_{A}$. The obtained results can be applied and expanded to the stability for the difference type's functional equation which consists of the (hyperbolic) secant, cosecant, logarithmic functions.
\end{abstract}

AMS Subject Classification 2010: 39B82, 39B52

Keywords Stability, Superstability, Cosine Functional Equation, Sine Functional Equation, Trigonometric Functional Equation

\section{Introduction}

In 1940, Ulam [24] conjectured the stability problem of the functional equation. Next year, Hyers [13] obtained partial answer for the case of additive mapping in this problem. [12].

Thereafter this problem was improved by Bourgin [9] in 1949, Aoki [4] in 1950, Th. M. Rassias [23] in 1978, and Găvruta

D’Alembert [1] in 1769(see, Kannappen book [15]) had introduced the cosine functional equation

$$
f(x+y)+f(x-y)=2 f(x) f(y),
$$

which superstability is proved by Baker [7] in 1980 .

Baker's result is generalized by Badora [5] in 1998 to a noncommutative group by using of the Kannappen condition [14]: $f(x+y+z)=f(x+z+y)$, and it again is improved by Badora and Ger [6] in 2002 under the condition $\mid f(x+y)+f(x-$ $y)-2 f(x) f(y) \mid \leq \varphi(x)$ or $\varphi(y)$. 
The cosine(d'Alembert) equation (C) is generalized to the following:

$$
\begin{aligned}
& f(x+y)+f(x-y)=2 f(x) g(y), \\
& f(x+y)+f(x-y)=2 g(x) f(y),
\end{aligned}
$$

which $\left(w f_{g}\right)$ is called by the Wilson equation.

The superstability of the cosine type equations $\left.(\overline{\mathbf{C}}), \sqrt{w f_{g}}\right)$ and $\left(\overline{w g_{f}}\right)$ is founded in Badora, Ger, Kannappan, and Kim ([8, 16, 19, ?]).

In 1983, Cholewa [10] investigated the superstability of the sine functional equation

$$
f(x) f(y)=f\left(\frac{x+y}{2}\right)^{2}-f\left(\frac{x-y}{2}\right)^{2} .
$$

His result was improved by $\operatorname{Kim}([18,20])$ in the following generalized sine functional equation

$$
g(x) h(y)=f\left(\frac{x+y}{2}\right)^{2}-f\left(\frac{x-y}{2}\right)^{2} .
$$

D'Alembert [1] studied the solution for the difference type's functional equation $f(x+y)-f(x-y)=2 f(x) g(y)$.

Kim [22] also proved the stability of functional equation

$$
f(x+y)-f(x-y)=\lambda \cdot h(x) k(y), \quad \lambda: \text { constant }
$$

Kim[17] has studied the superstability of the Pexider-type trigonometric functional equation

$$
f(x+y) \pm g(x-y)=\lambda f(x) g(y) .
$$

Fassi, Kabbaj, and Kim in [11] also obtained the superstability of cosine functional equation from $C_{f g h k}^{\lambda}$.

$$
f(x+y)+g(x-y)=\lambda h(x) k(y) .
$$

A trigonometric functional equation: $\cos (x+y)-\sin (x-y)=(\cos x-\sin x)(\cos y+\sin y)$ causes the functional equation $f(x+y)-g(x-y)=h(x) k(y)$.

The present work continues the study for the stability and solution of the sine function and Wilson equation from the following Pexider type functional equation

The aim of this paper is to study for the solution and the stability of the sine function and Wilson equation from the Pexider type's trigonometric functional equation:

$$
f(x+y)-g(x-y)=\lambda \cdot h(x) k(y), \quad \lambda: \text { constant } .
$$

under the conditions $\|f(x+y)-g(x-y)-\lambda h(x) k(y)\| \leq \varphi(x)$ or $\varphi(y)$ on an unital commutative normed algebra $(A,\|\cdot\|)$.

The obtained stability results for $\left[T_{f g h k}^{\lambda}\right.$ can be applied to the hyperbolic trigonometric functions, several exponential functions, and Jensen equation as follows:

$$
\begin{gathered}
\sin (x+y)-\cos (x-y)=(\sin x-\cos x)(\cos y-\sin y) \\
\cosh (x+y)-\sinh (x-y)=[\cosh (x)-\sinh (x)][\cosh (y)+\sinh (y)] \\
\cosh (x+y)-\cosh (x-y)=2 \sinh (x) \sinh (y) \\
\sinh (x+y)-\sinh (x-y)=2 \cosh (x) \sinh (y) \\
\sinh ^{2}\left(\frac{x+y}{2}\right)-\sinh ^{2}\left(\frac{x-y}{2}\right)=\sinh (x) \sinh (y) \\
e^{x+y}-e^{x-y}=2 \frac{e^{x}}{2}\left(e^{y}-e^{-y}\right)=2 e^{x} \cosh (y) \\
n(x+y)-n(x-y)=2 n y: \text { for } f(x)=n x .
\end{gathered}
$$

When $(\mathrm{G},+)$ relates to the sine functional equation $(S)$, it is a 2-divisible Abelian group, but it is not related to $(S)$, then it is a noncommutative semigroup.

Let $\mathbb{C}$ be a the set of complex numbers, and $\mathbb{C}^{*}=\mathbb{C} \backslash\{0\}, V$ is a vector space. $A$ is an unital commutative normed algebra with unit $1_{A}$, and $a^{-1}$ is an invertible element of $0 \neq a \in A$ (i.e $a^{-1} a=a a^{-1}=1_{A}$ ). And also $\varepsilon, \lambda>0$ be a constant, $\varphi: G \rightarrow[0, \infty)$ be a function.

Let us denote the cosine type equation, sine type equation, Wilson type equation, and trigonometric type equation as following:

$$
f(x+y)+f(x-y)=\lambda f(x) f(y)
$$




$$
\begin{aligned}
& f(x+y)-f(x-y)=\lambda f(x) f(y) \\
& h(x+y)+h(x-y)=\lambda h(x) k(y) \\
& h(x+y)-h(x-y)=\lambda h(x) k(y) .
\end{aligned}
$$

\section{Superstability of the Wilson and the sine functional equation.}

In this section, we will investigate the superstability of the Wilson and the sine functional equation from the mixed trigonometric functional equation $T_{f g h k}^{\lambda}$,

Theorem 1. Assume that $f, g, h, k: G \times G \rightarrow A$ satisfy the inequality

$$
\|f(x+y)-g(x-y)-\lambda h(x) k(y)\| \leq \varphi(x) \quad \forall x, y \in G .
$$

Suppose that there exists a sequence $\left\{y_{n}\right\}$ in $G$ such that $\lim _{n \rightarrow \infty}\left\|k\left(y_{n}\right)^{-1}\right\|=0$.

Then, (i) $h$ satisfies

$$
h(x+y)+h(x-y)=\lambda h(x) l_{k}(y) \quad \forall x, y \in G,
$$

in which, $l_{k}: G \rightarrow A$ is an even function such that $l_{k}(0)=2 \lambda^{-1}$.

(ii) $h$ satisfies $(S)$ under one of the cases $h(0)=0$ or $f(x)=g(-x)$.

(iii) In particular, if $k$ satisfies $\sqrt{C^{\lambda}}$, then $h$ and $k$ satisfies $\left(W^{\lambda} h_{k}\right)$.

Proof. (i) From assumption, we can choose $\left\{y_{n}\right\}$ such that $\left\|k\left(y_{n}\right)^{-1}\right\| \rightarrow 0$ as $n \rightarrow \infty$.

Putting $y=y_{n}$ (with $n \in \mathbb{N}$ ) in $(1)$, we have

$$
\left\|f\left(x+y_{n}\right)-g\left(x-y_{n}\right)-\lambda h(x) k\left(y_{n}\right)\right\| \leq \varphi(x)
$$

for all $x, y_{n} \in G$. Then

$$
\begin{aligned}
\|\left(f\left(x+y_{n}\right)-\right. & \left.g\left(x-y_{n}\right)\right) k\left(y_{n}\right)^{-1}-\lambda h(x) \| \\
& =\left\|\left(f\left(x+y_{n}\right)-g\left(x-y_{n}\right)-\lambda h(x) k\left(y_{n}\right)\right) k\left(y_{n}\right)^{-1}\right\| \\
& \leq\left\|f\left(x+y_{n}\right)-g\left(x-y_{n}\right)-\lambda h(x) k\left(y_{n}\right)\right\|\left\|k\left(y_{n}\right)^{-1}\right\| \\
& \leq\left\|k\left(y_{n}\right)^{-1}\right\| \varphi(x)
\end{aligned}
$$

for all $y \in G$. As $n \rightarrow \infty$ in (2), we get

$$
h(x)=\lambda^{-1} \lim _{n \rightarrow \infty}\left[f\left(x+y_{n}\right)-g\left(x-y_{n}\right)\right] k\left(y_{n}\right)^{-1}
$$

for all $y \in G$.

Replacing $y$ by $y+y_{n}$ in (1), we obtain

$$
\left\|f\left(x+\left(y+y_{n}\right)\right)-g\left(x-\left(y+y_{n}\right)\right)-\lambda h(x) k\left(y+y_{n}\right)\right\| \leq \varphi(x),
$$

for all $x, y, y_{n} \in V$.

Substituting $y$ by $-y+y_{n}$ in (1), we find

$$
\left\|f\left(x+\left(-y+y_{n}\right)\right)-g\left(x-\left(-y+y_{n}\right)\right)-\lambda h(x) k\left(-y+y_{n}\right)\right\| \leq \varphi(x),
$$

for all $x, y \in G$.

Then

$$
\begin{array}{r}
\| f\left(x+\left(y+y_{n}\right)\right)-g\left(x-\left(y+y_{n}\right)\right)-\lambda h(x) k\left(y+y_{n}\right)+f\left(x+\left(-y+y_{n}\right)\right) \\
-g\left(x-\left(-y+y_{n}\right)\right)-\lambda h(x) k\left(-y+y_{n}\right) \| \leq 2 \varphi(x)
\end{array}
$$

for all $x, y \in G$. 
This implies that

$$
\begin{aligned}
& \|\left[f\left((x+y)+y_{n}\right)-g\left((x+y)-y_{n}\right)\right] k\left(y_{n}\right)^{-1}+\left[f\left((x-y)+y_{n}\right)-g\left((x-y)-y_{n}\right)\right] k\left(y_{n}\right)^{-1} \\
& \quad-\lambda h(x)\left[k\left(y+y_{n}\right)+k\left(-y+y_{n}\right)\right] k\left(y_{n}\right)^{-1} \| \\
& =\|\left[f\left((x+y)+y_{n}\right)-g\left((x+y)-y_{n}\right)-\lambda h(x) k\left(y+y_{n}\right)\right. \\
& \left.\quad+f\left((x-y)+y_{n}\right)-g\left((x-y)-y_{n}\right)-\lambda h(x) k\left(-y+y_{n}\right)\right] k\left(y_{n}\right)^{-1} \| \\
& \leq\left\|k\left(y_{n}\right)^{-1}\right\| 2 \varphi(x)
\end{aligned}
$$

for all $x, y \in G$.

We notice that the right-hand side converges to zero as $n \rightarrow \infty$. So we can define a limit function $l_{k}: G \rightarrow F$ as follows

$$
l_{k}(y):=\lim _{n \rightarrow \infty} \frac{k\left(y+y_{n}\right)+k\left(-y+y_{n}\right)}{\lambda \cdot k\left(y_{n}\right)^{-1}}
$$

for all $y \in G$, then $l_{k}(0)=2 \lambda^{-1}$ and $l_{k}$ is an even function. Letting $n \rightarrow \infty$ in (7), we see from (3) that

$$
h(x+y)+h(x-y)=\lambda h(x) l_{k}(y) \quad \forall x, y \in G,
$$

as desired.

(ii-1) Applying the case $h(0)=0$ in $(9)$, it implies that $h$ is odd.

Putting $y=x$ in 9 , we get the equation

$$
h(2 x)=\lambda \cdot h(x) l_{k}(x), \quad x \in G .
$$

Keeping this odd in mind, by means of (9) and (10), we infer the equality

$$
\begin{aligned}
h(x+y)^{2}-h(x-y)^{2} & =\lambda h(x) l_{k}(y)[h(x+y)-h(x-y)] \\
& =\lambda h(x) l_{k}(y)[h(y+x)+h(y-x)] \\
& =\lambda h(x) l_{k}(y) \cdot \lambda h(y) l_{k}(x) \\
& =\lambda h(x) l_{k}(x) \cdot \lambda h(y) l_{k}(y) \\
& =h(2 x) \cdot h(2 y) .
\end{aligned}
$$

This, in return, leads to the equation

$$
h(x+y)^{2}-h(x-y)^{2}=h(2 x) h(2 y)
$$

valid for all $x, y \in G$ which, in the light of the unique 2-divisibility of $G$, states nothing else but $(S)$.

(ii-2) For the case $f(x)=g(-x)$, It is enough to show that $h(0)=0$. Suppose that this is not the case.

Putting $x=0$ in 1 , due to $h(0) \neq 0$ and $f(y)=g(-y)$, we obtain the inequality

$$
\|k(y)\| \leq \frac{\varphi(0)}{\lambda \cdot\|h(0)\|}, \quad y \in G .
$$

This inequality means that $k$ is globally bounded, which is a contradiction. Thus, the claimed $h(0)=0$ holds.

(iii) In the case $k$ satisfies $\sqrt{C^{\lambda}}$, the limit $l_{k}$ states nothing else but $k$ from $(8)$, so $h$ and $k$ validate a required equation $W^{\lambda} h_{k}$.

Theorem 2. Assume that $f, g, h, k: G \times G \rightarrow A$ satisfy the inequality

$$
\|f(x+y)-g(x-y)-\lambda h(x) k(y)\| \leq \varphi(y) \quad \forall x, y \in G .
$$

Suppose that there exists a sequence $\left\{x_{n}\right\}$ in $G$ such that $\lim _{n \rightarrow \infty}\left\|h\left(x_{n}\right)^{-1}\right\|=0$.

Then, (i) $k$ satisfies

$$
k(x+y)+k(x-y)=\lambda k(x) l_{h}(y),
$$

in which, $l_{h}$ is an even function such that $l_{h}(0)=2 \lambda^{-1}$.

(ii) $k$ satisfies $\sqrt{S}$ ) under one of the cases $k(0)=0$ or $f(x)=g(x)$,

(iii) In particular, if $h$ satisfies $C^{\lambda}$, then $k$ and $h$ satisfies

$$
k(x+y)+k(x-y)=\lambda \cdot k(x) h(y) .
$$


Proof. Let us choose $\left\{x_{n}\right\}$ in $G$ such that $\left.\lim _{n \rightarrow \infty} \| h\left(x_{n}\right)\right)^{-1} \|=0$. that

Taking $x=x_{n}$ (with $n \in \mathbb{N}$ ) in $\left[12\right.$, dividing both sides by $\left\|\lambda \cdot h\left(x_{n}\right)\right\|$, and passing to the limit as $n \rightarrow \infty$, we obtain

$$
k(y)=\lambda^{-1} \lim _{n \rightarrow \infty}\left[f\left(x_{n}+y\right)-g\left(x_{n}-y\right)\right] h\left(x_{n}\right)^{-1}
$$

for all $x_{n}, y \in G$

Replace $(x, y)$ by $\left(x_{n}+y, x\right)$ and replace $(x, y)$ by $\left(x_{n}-y, x\right)$ in 12$)$. Thereafter we go through same procedure as the equations (4) (7) of Theorem 1, i.e., add the above two inequalities, and dividing by $\lambda \cdot h\left(x_{n}\right)$, then it gives us the existence of a limit function

$$
l_{h}(y):=\lim _{n \rightarrow \infty} \frac{h\left(x_{n}+y\right)+h\left(x_{n}-y\right)}{\lambda \cdot h\left(x_{n}\right)},
$$

where the function $l_{h}: G \rightarrow \mathbb{C}$ satisfies the equation

$$
k(x+y)+k(x-y)=\lambda \cdot k(x) l_{h}(y) \quad \forall x, y \in G
$$

as desired.

The process of the remainders (ii) and (iii) are achieved by following the same steps as Theorem 1 .

The following corollaries follow immediate from the Theorems 1 , 2 .

Corollary 3. Assume that $f, g, h, k: G \times G \rightarrow A$ satisfy the inequality

$$
\|f(x+y)-g(x-y)-\lambda h(x) k(y)\| \leq\left\{\begin{array}{l}
(i) \varphi(x) \\
(i i) \varphi(y) \\
(i i i) \varepsilon
\end{array}\right.
$$

(a) Suppose that there exists a sequence $\left\{y_{n}\right\}$ in $G$ such that

$$
\lim _{n \rightarrow \infty}\left\|k\left(y_{n}\right)^{-1}\right\|=0 .
$$

Then,

(i) $h$ satisfies $\left[W^{\lambda} h_{l_{k}}\right.$ with even function $l_{k}$ s.t. $l_{k}(0)=2 \lambda^{-1}$.

In particular, if $k$ satisfies $\left(C^{\lambda}\right)$, then $h$ and $k$ satisfies $\left(W^{\lambda} h_{k}\right)$.

(ii) $h$ satisfies $(S)$ under one of the cases $h(0)=0$ or $f(x)=g(-x)$.

(b) Suppose that there exists a sequence $\left\{x_{n}\right\}$ in $G$ such that

$$
\lim _{n \rightarrow \infty}\left\|h\left(x_{n}\right)^{-1}\right\|=0 .
$$

Then,

(i) $k$ satisfies $\left(W^{\lambda} k_{l_{h}}\right)$ with even function $l_{h}$ s.t. $l_{h}(0)=2 \lambda^{-1}$.

In particular, if $h$ satisfies $\left[C^{\lambda}\right.$, then $k$ and $h$ satisfies $\left(W^{\lambda} k_{h}\right.$.

(ii) $k$ satisfies $\sqrt{S})$ under one of the cases $k(0)=0$ or $f(x)=g(x)$.

Remark 1. In all results, we can obtain more corollaries from setting as the following.

(i) Replacing ordered for 4 functions: $f, g, h, k$.

(ii) Also letting $\varphi(x)=\varphi(y)=\varepsilon$ : positive constant, letting $\lambda=2$, it imply some corollaries.

Namely, from (i) and (ii), it imply the superstability of the cosine functional equation or the Wilson functional equation, respectively. (See R. Badora [5], R. Badora and R. Ger [6], J.A. Baker [5], G.H. Kim ( [17, 19, ?]), Pl. Kannappan and G.H. $\operatorname{Kim}[16]$. 


\section{Representation of Solution for the Wilson type equation.}

The solution of the Wilson equation $\left(w f_{g}\right)$ was investigated in papers ( d'Alembert [1], Aczel [2], Aczel and Dhombres [3], Kannappan ([14, 15]), Kannappan and G.H. Kim [16]).

In complex analysis, the trigonometric function can be represented by the exponential function. Hence, from the obtained results in Sections 2 , the explicit solution of Wilson type equation $\left(\overline{W f_{g}}\right)$ is estimated and can be found by direct calculations and can also be obtained directly from Lemma.

Let us find the solution for the trigonometric equations contained cosine(d'Alembert), sine, Wilson type equations. $W f_{g}$. And let us investigate a representation of a solution for them.

It is easy to verify shows that the following lemma holds.

Lemma 4. Let $f, g: G \times G \rightarrow \mathbb{C}^{*}$ satisfy the Wilson type equation

$$
\begin{aligned}
& f(x+y)+f(x-y)=\lambda f(x) g(y), \\
& f(x+y)+f(x-y)=\lambda g(x) f(y),
\end{aligned}
$$

Then, $g$ satisfies $C^{\lambda}$, and $g, f$ are given by

$$
g(x)=\frac{E(x)+E(-x)}{\lambda}, \quad f(x)=c(E(x)-E(-x))+\frac{d}{\lambda}(E(x)+E(-x)),
$$

where $c, d \in \mathbb{C}$, and $E: G \rightarrow \mathbb{C}^{*}$ is a homomorphism.

Letting $\lambda=2$ in the case(ii) of Remark 1 in Section 2, then we obtain the stability of Wilson type equations. $w f_{g}$ and $w f_{g}$ ) from the stability results of $T_{f g h k}^{\lambda}$. It's solution's types be represented as following results.

Theorem 5. Assume that $f, g, h, k: G \times G \rightarrow \mathbb{C}^{*}$ satisfy the inequality

$$
|f(x+y)-g(x-y)-\lambda h(x) k(y)| \leq \varphi(x) \quad \forall x, y \in G .
$$

If $k$ fails to be bounded, then

(i) $h$ and $l_{k}$ satisfy $\left(W^{\lambda} h_{l_{k}}\right.$. In particular, if $k$ satisfies $(C)$, then $h$ and $k$ satisfies $\left(W^{\lambda} h_{k}\right)$,) and $h, k$ are given by

$$
k(x)=\frac{E(x)+E(-x)}{\lambda}, \quad h(x)=c(E(x)-E(-x))+\frac{d}{\lambda}(E(x)+E(-x)),
$$

where $c, d \in \mathbb{C}$, and $E: G \rightarrow \mathbb{C}^{*}$ is a homomorphism.

(ii) $h$ satisfies $(S)$ under one of the cases $h(0)=0$ or $f(-x)=-g(x)$, and $h$ is of the form

$$
h(x)=A(x) \text { or } h(x)=c(E(x)-E(-x)),
$$

where $A: G \rightarrow C$ is an additive function, $c \in \mathbb{C}, E$ is as in (i).

Proof. The proof of the Corollary is enough from Theorem 1 except for the solution. However, they are immediate from the following:

(i) Appealing to the solutions of the Wilson equation $\left(w f_{g}\right]$ in paper [14] and [15](theorem 3.41, pp. 148) (see also [2, 3]), then the given explicit solutions are taken from it.

(ii) Appealing to the solutions of $(S)$ in paper ([15], theorem 3.44, pp. 153) (see also[14]), the explicit shapes of $h$ are as stated in the statement of the theorem. This completes the proof of the Corollary.

Corollary 6. Assume that $f, g, h, k: G \times G \rightarrow \mathbb{C}^{*}$ satisfy the inequality

$$
|f(x+y)-g(x-y)-\lambda h(x) k(y)| \leq \varphi(y) \quad \forall x, y \in G .
$$

If $h$ fails to be bounded, then

(i) $k$ and $l_{h}$ satisfy $\left(W^{\lambda} k_{l_{h}}\right.$. In particular, if $h$ satisfies $(C)$, then $k$ and $h$ satisfies $\sqrt{W^{\lambda} k_{h}}$, and $k, h$ are given by

$$
h(x)=\frac{E(x)+E(-x)}{\lambda}, \quad k(x)=c(E(x)-E(-x))+\frac{d}{\lambda}(E(x)+E(-x)),
$$

where $c, d \in \mathbb{C}$, and $E: G \rightarrow \mathbb{C}^{*}$ is a homomorphism.

(ii) $k$ satisfies $[S]$ under one of the cases $h(0)=0$ or $f(-x)=-g(x)$, and $k$ is of the form

$$
k(x)=A(x) \quad \text { or } k(x)=c(E(x)-E(-x)),
$$

where $A: G \rightarrow C$ is an additive function, $c \in \mathbb{C}, E$ is as in (i). 
Corollary 7. Suppose that $f, g, h, k: G \rightarrow \mathbb{C}^{*}$ satisfy the inequality

$$
|f(x+y)-g(x-y)-\lambda h(x) k(y)| \leq \varphi(x), \text { or } \varphi(y), \text { or } \varepsilon \quad \forall x, y \in G .
$$

(a)If $k$ fails to be bounded, then

(i) $h$ and $l_{k}$ satisfy $\left(W^{\lambda} h_{l_{k}}\right)$. In particular, if $k$ satisfies $(\mathbb{C})$, then $h$ and $k$ satisfies $\left(W^{\lambda} h_{k}\right.$, ,) and $h, k$ are given by

$$
k(x)=\frac{E(x)+E(-x)}{\lambda}, \quad h(x)=c(E(x)-E(-x))+\frac{d}{\lambda}(E(x)+E(-x)),
$$

where $c, d \in \mathbb{C}$, and $E: G \rightarrow \mathbb{C}^{*}$ is a homomorphism.

(ii) $h$ satisfies $[S]$ under one of the cases $h(0)=0$ or $f(-x)=-g(x)$, and $h$ is of the form

$$
h(x)=A(x) \quad \text { or } h(x)=c(E(x)-E(-x)),
$$

where $A: G \rightarrow C$ is an additive function, $c \in \mathbb{C}, E$ is as in (i).

(b) If $h$ fails to be bounded, then

(i) $k$ and $l_{h}$ satisfy $\left[W^{\lambda} k_{l_{h}}\right.$. In particular, if $h$ satisfies $(C)$, then $k$ and $h$ satisfies $\left(W^{\lambda} k_{h}\right]$,) and $k, h$ are given by

$$
h(x)=\frac{E(x)+E(-x)}{\lambda}, \quad k(x)=c(E(x)-E(-x))+\frac{d}{\lambda}(E(x)+E(-x)),
$$

where $c, d \in \mathbb{C}$, and $E: G \rightarrow \mathbb{C}^{*}$ is a homomorphism.

(ii) $k$ satisfies $(S)$ under one of the cases $h(0)=0$ or $f(-x)=-g(x)$, and $k$ is of the form

$$
k(x)=A(x) \quad \text { or } k(x)=c(E(x)-E(-x)),
$$

where $A: G \rightarrow C$ is an additive function, $c \in \mathbb{C}, E$ is as in (i).

Remark 2. Replacing ordered for 4 functions as Remark 1 can be implies 15 equations. Therefore, as like the above corollaries 6, 7), we can obtain additionally the results of numbers $(14$ (equations $) \times 3(\varphi(x), \varphi(y), \varepsilon))$. Some, which excepted the explicit solutions represented by composition of a homomorphism, in the obtained results are found in papers $([6,12,16,17,19,22])$.

\section{Application to the Banach space}

In all results obtained in Section 2, the range of functions on the Abelian group can be extended to the semisimple commutative Banach space. We will represent only for the main equation $T_{f g h k}^{\lambda}$.

Theorem 8. Let $(E,\|\cdot\|)$ be a semisimple commutative Banach space. Assume that $f, g, h, k: G \rightarrow E$ satisfy one of each inequalities

$$
\|f(x+y)-g(x-y)-\lambda \cdot h(x) k(y)\| \leq\left\{\begin{array}{l}
(a) \varphi(x), \\
(b) \varphi(y),
\end{array} \quad \forall x, y \in G .\right.
$$

For an arbitrary linear multiplicative functional $x^{*} \in E^{*}$,

(a) Suppose that $x^{*} \circ k$ fails to be bounded, then

(i) $h$ satisfies $\sqrt{W^{\lambda} h_{l_{k}}}$ with an even function $l_{k}$ such that $l_{k}(0)=2 \lambda^{-1}$. In particular, if $k$ satisfies $C^{\lambda}$, then $h$ and $k$ satisfies the $\left(W^{\lambda} h_{k}\right)$.

(ii) $h$ satisfies $\sqrt{S}$ ) under one of the cases $\left(x^{*} \circ h\right)(0)=0$ or $\left(x^{*} \circ f\right)(x)=-\left(x^{*} \circ g\right)(x)$.

(b) Suppose that $x^{*} \circ h$ fails to be bounded, then

(i) $k$ satisfies $\sqrt{W^{\lambda} k_{l_{h}}}$ with an even function $l_{h}$ such that $l_{h}(0)=2 \lambda^{-1}$. In particular, if $h$ satisfies $\sqrt{C^{\lambda}}$, then $k$ and $h$ satisfies the $W^{\lambda} k_{h}$.

(ii) $k$ satisfies $\sqrt{S})$ under one of the cases $\left(x^{*} \circ k\right)(0)=0$ or $\left(x^{*} \circ f\right)(-x)=-\left(x^{*} \circ g\right)(x)$.

Proof. (a)(i). Assume that 16 holds and arbitrarily fixes a linear multiplicative functional $x^{*} \in E^{*}$. As is well known, we have $\left\|x^{*}\right\|=1$, hence, for every $x, y \in G$, we have

$$
\begin{aligned}
\varphi(x) & \geq\|f(x+y)-g(x-y)-\lambda \cdot h(x) k(y)\| \\
& =\sup _{\left\|y^{*}\right\|=1}\left|y^{*}(f(x+y)-g(x-y)-\lambda \cdot h(x) k(y))\right| \\
& \geq\left|x^{*}(f(x+y))-x^{*}(g(x-y))-\lambda \cdot x^{*}(h(x)) x^{*}(k(y))\right|,
\end{aligned}
$$


which states that the superpositions $x^{*} \circ f, x^{*} \circ g, x^{*} \circ h$ and $x^{*} \circ k$ yield a solution of inequality (1) in Theorem 1 Since, by assumption, the superposition $x^{*} \circ k$ is unbounded, an appeal to Theorem 1 shows that the two results hold.

First, the superposition $x^{*} \circ h$ solves $\sqrt{W^{\lambda} h_{l_{k}}}$ with $x^{*} \circ l_{k}$ that is

$$
\left(x^{*} \circ h\right)(x+y)-\left(x^{*} \circ h\right)(x-y)=\lambda\left(x^{*} \circ h\right)(x)\left(x^{*} \circ l_{k}\right)(y) \text {. }
$$

Since $x^{*}$ is a linear multiplicative functional, we get

$$
x^{*}\left(h(x+y)-h(x-y)-\lambda \cdot h(x) l_{k}(y)\right)=0 .
$$

Hence a unrestricted choice of $x^{*}$ implies that

$$
h(x+y)-h(x-y)-\lambda \cdot h(x) l_{k}(y) \in \bigcap\left\{\operatorname{ker} x^{*}: x^{*} \in E^{*}\right\}
$$
with $l_{k}$

Since the space $E$ is semisimple, $\bigcap\left\{\operatorname{ker} x^{*}: x^{*} \in E^{*}\right\}=0$, which means that $h$ satisfies the claimed equation $W^{\lambda} h_{l_{k}}$

In particular, if $k$ satisfies $\sqrt{C^{\lambda}}$, then $l_{k}$ implies $k$, hence $\left[W^{\lambda} h_{k}\right.$ holds.

(a)(ii). By assumption, the superposition $x^{*} \circ k$ is unbounded, an appeal to Theorem 1 shows that the two results hold.

First, in case $\left(x^{*} \circ h\right)(0)=0$, the superposition $x^{*} \circ h$ solves $(S)$, that is

$$
\left(x^{*} \circ h\right)\left(\frac{x+y}{2}\right)^{2}-\left(x^{*} \circ h\right)\left(\frac{x-y}{2}\right)^{2}=\lambda\left(x^{*} \circ h\right)(x)\left(x^{*} \circ h\right)(y) .
$$

This means by a linear multiplicativity of $x^{*}$ that

$$
\mathcal{D} S_{h}^{\lambda}(x, y):=h\left(\frac{x+y}{2}\right)^{2}-h\left(\frac{x-y}{2}\right)^{2}-\lambda h(x) h(y)
$$

falls into the kernel of $x^{*}$. Namely, $x^{*}\left(\mathcal{D} C_{h k}^{\lambda}(x, y)\right)=0$.

$$
x^{*}\left(\mathcal{D} S_{h}^{\lambda}(x, y)\right)=0 .
$$

Hence a unrestricted choice of $x^{*}$ implies that

$$
\mathcal{D} S_{h}^{\lambda}(x, y) \in \bigcap\left\{\operatorname{ker} x^{*}: x^{*} \in E^{*}\right\}
$$

Since the space $E$ is semisimple, $\bigcap\left\{\operatorname{ker} x^{*}: x^{*} \in E^{*}\right\}=0$, which means that $h$ satisfies the claimed equation $\sqrt{S \text {. }}$.

For second case $\left(x^{*} \circ f\right)(x)=-\left(x^{*} \circ g\right)(x)$, it is enough to show that $\left(x^{*} \circ h\right)(0)=0$, which can be easily check as Theorem 1 Hence, the proof (ii) of (a) is completed.

(b) The case $\varphi(y)$ is same due to Theorem 2 as like the case (a).

Theorem 9. Let $(E,\|\cdot\|)$ be a semisimple commutative Banach space. Assume that $f, g, h, k: G \rightarrow E$ satisfy one of each inequalities

$$
\|f(x+y)-g(x-y)-\lambda \cdot h(x) k(y)\| \leq \varphi(x) \text {, or } \varphi(y), \text { or } \varepsilon \quad \forall x, y \in G .
$$

for all $x, y \in G$. For an arbitrary linear multiplicative functional $x^{*} \in E^{*}$,

(a) Suppose that $x^{*} \circ k$ fails to be bounded, then

(i) $h$ satisfies $\left[W^{\lambda} h_{l_{k}}\right)$ with an even function $l_{k}$ such that $l_{k}(0)=2 \lambda^{-1}$. In particular, if $k$ satisfies $\left.\sqrt{C^{\lambda}}\right]$, then $h$ and $k$ satisfies the $W^{\lambda} h_{k}$.

(ii) $h$ satisfies $[S)$ under one of the cases $\left(x^{*} \circ h\right)(0)=0$ or $\left(x^{*} \circ f\right)(x)=-\left(x^{*} \circ g\right)(x)$,

(b) Suppose that $x^{*} \circ h$ fails to be bounded, then

(i) $k$ satisfies $\sqrt{W^{\lambda} k_{l_{h}}}$ with an even function $l_{h}$ such that $l_{h}(0)=2 \lambda^{-1}$. In particular, if $h$ satisfies $\left.\overline{C^{\lambda}}\right]$, then $k$ and $h$ satisfies the $W^{\lambda} k_{h}$.

(ii) $k$ satisfies $[S]$ under one of the cases $\left(x^{*} \circ k\right)(0)=0$ or $\left(x^{*} \circ f\right)(-x)=-\left(x^{*} \circ g\right)(x)$.

\section{Conclusion}

In this paper, we proved the superstability of the sine functional equation and the Wilson equation from the Pexider type difference functional equation $f(x+y)-g(x-y)=\lambda \cdot h(x) k(y)$, which is the mixed equation represented by the sine, the cosine, the tangent, the hyperbolic trigonometric functions, and exponential functions.

Also, we obtained additionally that the Wilson equation and the cosine functional equation in the obtained results can be represented by the compositions of a homomorphism $E(x)$ such that $k(x)=\frac{E(x)+E(-x)}{\lambda}, h(x)=c(E(x)-E(-x))+$ $\frac{d}{\lambda}(E(x)+E(-x)), \quad h(x)=A(x)$, or $h(x)=c(E(x)-E(-x))$. 


\section{Acknowledgment}

This work was supported by Kangnam University Research Grant in 2018.

\section{REFERENCES}

[1] J. d'Alembert, Memoire sur les Principes de Mecanique, Hist. Acad. Sci. Paris, (1769), 278-286

[2] J. Aczel, Lectures on Functional Equations and their Applications, Academic Press, New York, 1966.

[3] J. Aczel, J. Dhombres, Functional Equations in Several Variables, Cambridge University Press, Cambridge, 1988.

[4] T. Aoki, On the stability of the linear transformation in Banach spaces, J. Math. Soc. Japan, 2 (1950), 64-66.

[5] R. Badora, On the stability of cosine functional equation, Rocznik Naukowo-Dydak., Prace Mat., 15 (1998), 1-14.

[6] R. Badora and R. Ger, On some trigonometric functional inequalities, Functional Equations- Results and Advances, (2002), 3-15.

[7] J. A. Baker, The stability of the cosine equation, Proc. Amer. Math. Soc., 80 (1980), 411-416.

[8] B. Bouikhalene, E. Elqorachi, and J. M. Rassias, The superstability of d'Alembert's functional equation on the Heisenberg group, Appl. Math. Lett., vol. 23 (2010), 105-109.

[9] D. G. Bourgin, Approximately isometric and multiplicative transformations on continuous function rings, Duke Math. J., 16, (1949), 385-397.

[10] P.W. Cholewa, The stability of the sine equation, Proc. Amer. Math. Soc., 88 (1983). 631-634.

[11] I.E. Fassi, S. Kabbaj, G.H. Kim, Superstability of a Pexider-type Trigonometric Functional Equation in Normed Algebras, Inter. J. Math. Anal., 9(58), (2015) 2839 - 2848

[12] P. Găvruta, On the stability of some functional equations, in : Th. M. Rassias and J. Tabor, Stability of mappings of Hyers-Ulam type, Hadronic Press, 1994, pp. 93-98.

[13] D. H. Hyers, On the stability of the linear functional equation, Proc. Natl. Acad. Sci. 27(1941), 222-224.

[14] Pl. Kannappan, The functional equation $f(x y)+f\left(x y^{-1}\right)=2 f(x) f(y)$ for groups, Proc. Amer. Math. Soc., 19 (1968), 69-74.

[15] Pl. Kannappan, Functional equations and inequailitis with applications, Springer, 2009.

[16] Pl. Kannappan and G. H. Kim, On the stability of the generalized cosine functional equations, Ann. Acad. Pedagog. Crac. Stud. Math., 1 (2001), 49-58.

[17] G. H. Kim, On the superstability of the pexider type trigonometric functional equation, Journal of Inequalities Applications, 2010 (2010), Doi:10.1155/2010/897123.

[18] G. H. Kim, On the Stability of the generalized sine functional equations, Acta Math. Sin., Engl. Ser. 25 (2009), 965-972

[19] G. H. Kim, The Stability of the d'Alembert and Jensen type functional equations, Jour. Math. Anal \& Appl., 325 (2007), 237-248.

[20] G. H. Kim, A Stability of the generalized sine functional equations, Jour. Math. Anal \& Appl., 331 (2007), 886-894.

[21] G. H. Kim, On the Stability of the Pexiderized trigonometric functional equation, Appl. Math. Compu., 203 (2008), 99-105

[22] G. H. Kim, Superstability of a generalized trigonometric functional equation, Nonlinear Functional Analysis and Applications, 24(2) (2019).

[23] Th. M. Rassias, On the stability of the linear mapping in Banach spaces, Proc. Amer. Math. Soc. 72(1978), 297-300.

[24] S. M. Ulam, "Problems in Modern Mathematics" Chap. VI, Science editions, Wiley, New York, 1964 